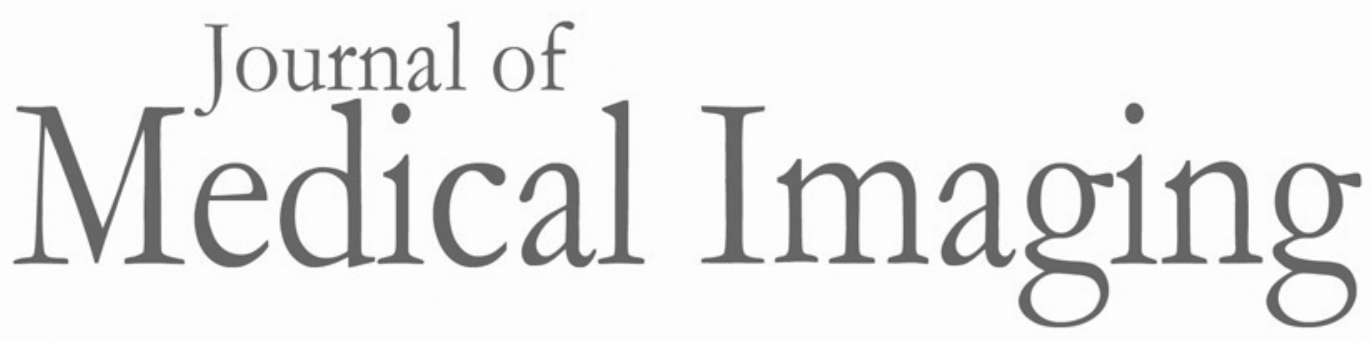

Medicallmaging.SPIEDigitalLibrary.org

\title{
Visions of Safety: Perspectives on Radiation Exposure and Risk in Medical Imaging
}

Ehsan Samei

Christoph Hoeschen 


\title{
Visions of Safety: Perspectives on Radiation Exposure and Risk in Medical Imaging
}

\author{
Ehsan Samei \\ Duke University \\ Departments of Radiology, Physics, Biomedical Engineering, and Electrical and Computer Engineering \\ Clinical Imaging Physics Group, Medical Physics Graduate Program, and \\ Carl E. Ravin Advanced Imaging Laboratories (RAI Labs) \\ Durham, North Carolina, United States
}

\section{Christoph Hoeschen}

Otto-von-Guericke University

Faculty for Electrical Engineering and Information Technology

Institute for Medical Technology

Magdeburg, Germany

Since the very early years of using ionizing radiation for medical diagnosis and therapy, it became obvious that beside the benefits of an accurate diagnosis or a successful treatment, ionizing radiation may also have harmful effects. Over many decades, there have been extensive scholarship and dialogue about how to deal with the associated risks and how to justify the use of ionizing radiation on humans for each application. Increased reliance on medical imaging in the last 15 years has brought these issues into a sharper focus. The increased life expectancy and increased social sensitivity to risk factors have further fueled the attention.

Historical grounding: While a main focus of medical imaging has always been the development and implementation of technology, even a few years after the discovery of the $x$-rays by Wilhelm Conrad Röntgen, it became evident that besides their beneficiary role, ionizing radiation can have hazardous effects. This motivated the physics-based description of radiation dose, resulting in the definition of various dose definitions and units including organ dose, dose equivalent, and effective dose. Over years, nonionizing imaging modalities [such as magnetic resonance imaging (MRI) and ultrasound] also established a valuable role in medicine. However, that did not and has not diminished the role of ionizing radiation-based medical imaging procedures, primarily due to their advantages in speed, robustness, and quantitation, especially in nuclear imaging exams and in CT. In fact, in the last two decades, these advantages, coupled with new applications, have led to increased utilization of ionizing radiation imaging exams.

Ethical responsibility in spite of ambiguity of risk: The science of radiation biology pertaining to the effects of ionizing radiation has been developed primarily based on retrospective analysis of survivors of Japanese atomic bombs and nuclear accidents. This mainly biologically driven and partly epidemiologically driven research provides answers to effects of ionizing radiation in the medium and high dose ranges, above at least $100 \mathrm{mSv}$. Most imaging doses are well below $100 \mathrm{mSv}$. Effects below $100 \mathrm{mSv}$ can be found in biological probes but are difficult to prove in whole organisms or in human cohorts. This has led to strong debate whether low levels of radiation associated with medical imaging have a detrimental effect. In spite of the debate, there remains a

(C) 2017 Society of Photo-Optical Instrumentation Engineers (SPIE) prevailing conviction, reflected by the International Commission on Radiological Protection (ICRP), that the potential detrimental effect even at low dose levels cannot be ruled out. And if there is a potential risk, one can argue that there is an ethical responsibility of the medical establishment that the radiation risk should be managed and minimized.

Professional efforts to manage imaging safety: In recent years, the above responsibility has led to rigor in justification of exams (whether an exam is needed in the first place), optimization of exams (whether the dose is appropriate for a given exam), and minimization of risk (managing population level dose considering the increased utilization). Prominent professional efforts to manage imaging safety include Image Gently and Image Wisely in the US, EuroSafe and EURAMED in Europe, AfroSafe in Africa, and other like campaigns. These initiatives aim to foster good practice and research on medical-related biological exposure effects, optimization of imaging procedures in terms of dose, and the implementation of methodologies proposed by radiation protection bodies intending to manage dose associated with medical application of ionizing radiation. These efforts are instrumental to create common ground and to form consensus for best practices.

In this issue: This special section aims to highlight the basic understandings of dose measurement, risk estimation, and optimization in medical imaging as well as the actual achievements in such areas to provide a comprehensive vision of safety in radiological imaging. The large number of manuscripts (13) in this special section indicates the importance and the increasing relevance of this subject. Topics covered include:

- Science of dose estimation (papers by Vijayan et al., Xiong et al., and Hoye et al.)

- Dose metrology (papers by Markovich et al. and Ford et al.)

- Summary of current practice (papers by Muhogora and Rehani and Berris et al.)

- Broad perspectives on dose management (papers by Jarvinen et al. and Rehani)

- Technological advances for dose reduction (papers by Hedgire et al. and Fu et al.) 
- Science of imaging optimization (papers by Samei et al. and Favazza et al.).

We are thankful to all the contributing authors who shared their valuable insights. Within these excellent manuscripts, the readers will find a broad range of science from quantification of dose, to its optimization balance with respect to image quality in adult and pediatric exams, to dose management through diagnostic reference levels, to techniques of dose reduction, to current status of practice in various parts of the world.

What's next? While the material in this special section is impressive, much still needs to be done. We hope that this issue inspires our community to tackle some of the most vexing and needed topics pertaining radiation safety. Below we include a few:

- Ethics of safety

- Mechanism of harm from radiation burden

- The relation between low-level dose and radiation risk, individually, biologically, and epidemiologically

- Risk and risk estimation in the context of uncertainty

- Modality-specific patient-specific dosimetry

- Inflated safety concerns for doses below 50-100 mSv

- Safety of overexposure versus under-diagnosis

- Imaging safety from clinical perspective

- Radiation safety in pediatric imaging across modalities

- Role of regulations in radiation safety
- Practical methods for risk mitigation by dose optimization

- Practical methods for risk mitigation by appropriateness justification of exams

We would like to encourage the readers of this special section to tackle these relevant topics. We hope papers corresponding to these topics will be published in the future issues of the Journal of Medical Imaging. The results will hopefully lead to a measurable impact on patient care throughout the world.

Ehsan Samei, PhD, is a tenured professor at Duke University, where he serves as the director of the Duke medical physics graduate program and clinical imaging physics. His interests include clinically relevant metrology of imaging quality and safety for optimum interpretive and quantitative performance. He strives to bridge the gap between scientific scholarship and clinical practice by 1) meaningful realization of translational research and 2) the actualization of clinical processes that are informed by scientific evidence. $\mathrm{He}$ is a diplomate of the American Board of Radiology, and a fellow of AAPM, SPIE, and AIMBE. He is also a distinguished Investigator of ARR and a councilor of NCRP.

Christoph Hoeschen, PhD, is full professor for medical technical systems at the Otto-von-Guericke University (OvGU) in Magdeburg, Germany. He headed a research unit at Helmholtz Zentrum München dealing with medical physics and especially diagnostics, which characterizes his interests (new diagnostic tools and their characterization including radiation protection). He holds a PhD from the OvGU. He belongs to the German radiation protection board and is founding chair of EURAMED platform. He is a senior member of SPIE and an associate editor of the Journal of Medical Imaging. 